\title{
The structural basis for deadenylation by the CCR4-NOT complex
}

\author{
Mark Bartlam ${ }^{1 凶}$, Tadashi Yamamoto ${ }^{2 凶}$ \\ 1 Tianjin Key Laboratory of Protein Science, College of Life Sciences, Nankai University, Tianjin 300071, China \\ 2 Division of Oncology, Institute of Medical Science, University of Tokyo, Minato-ku, Tokyo 108-8639, Japan \\ $\checkmark$ Correspondence: bartlam@nankai.edu.cn (M. Bartlam), tyamamot@ims.u-tokyo.ac.jp (T. Yamamoto) \\ Received April 21, 2010 Accepted May 5, 2010
}

\begin{abstract}
The CCR4-NOT complex is a highly conserved, multifunctional machinery controlling mRNA metabolism. Its components have been implicated in several aspects of mRNA and protein expression, including transcription initiation, elongation, mRNA degradation, ubiquitination, and protein modification. In this review, we will focus on the role of the CCR4-NOT complex in mRNA degradation. The complex contains two types of deadenylase enzymes, one belonging to the DEDD-type family and one belonging to the EEP-type family, which shorten the poly $(A)$ tails of mRNA. We will review the present state of structure-function analyses into the CCR4-NOT deadenylases and summarize current understanding of their roles in mRNA degradation. We will also review structural and functional work on the Tob/BTG family of proteins, which are known to interact with the CCR4-NOT complex and which have been reported to suppress deadenylase activity in vitro.
\end{abstract}

\section{INTRODUCTION}

The CCR4-NOT complex is highly and evolutionarily conserved among eukaryotes (Draper et al., 1995; Albert et al., 2000; Gavin et al., 2002). It has been found in two predominant "supercomplex" forms in vivo, with approximate molecular weights of 0.9-1.2 MDa and 1.9-2.0 MDa in yeast. The smaller core complex in Saccharomyces cerevisiae (Liu et al., 1998; Bai et al., 1999; Chen et al., 2001; Cui et al., 2008) was identified to contain five NOT proteins (Not1p to Not5p), Pop2p, Caf40p, Caf130p, Ccr4p and BTT1. A number of other proteins known to interact with Ccr4p, Pop2p or NOT proteins, such as Dhh1p Caf4p, Caf16p, Dbf2p and Mob1p, are believed to be part of the larger complex (Liu et al., 1997; Hata et al., 1998; Komarnitsky et al., 1998). In mammals, including humans, their homologs also form a similar multisubunit complex which plays a significant role in the regulation of several cellular machines (Mahadevan and Struhl, 1990; Seufert and Jentsch, 1990; Collart and Struhl, 1993, 1994; Moqtaderi et al., 1996; Lee et al., 1998; Oberholzer and Collart, 1998; Badarinarayana et al., 2000; Tucker et al., 2001; Albert et al., 2002; Deluen et al., 2002; Lenssen et al., 2002; Maillet and Collart, 2002; Tucker et al., 2002; Viswanathan et al., 2003).

Despite increasing understanding of the properties of the human CCR4-NOT complex, relatively little is known about its three-dimensional structure or its precise roles in the cell. The CCR4-NOT complex is implicated in a number of cellular functions, including several aspects of mRNA regulatory pathways (Denis and Chen, 2003; Collart and Timmers, 2004). The presence of human CNOT4 and yeast Not4p, which both harbour E3 ligase activity, places the CCR4-NOT complex in the protein ubiquitination/degradation pathways, although a recent analysis has suggested that the human CNOT4 does not stably interact with other human CCR4-NOT components and instead resides in a smaller $\sim 200 \mathrm{kDa}$ complex outside the CCR4-NOT "supercomplex" (Lau et al., 2009). Members of the CCR4-NOT complex are also associated with various functions in several species, either in the nucleus or the cytoplasm, including DNA repair and histone methylation in yeast, spindle positioning and regulation of microtubule length in Caenorhabditis elegans and spermatogenesis in mice.

Of the various functions with which the CCR4-NOT complex is associated, deadenylation has perhaps the greatest importance as it is crucial for gene expression and is involved in many biologic processes, from embryonic 
development to learning and memory (Goldstrohm and Wickens, 2008). Regulation of mRNA stability, translation and localization determines how much protein is produced during mRNA translation. Newly synthesized mRNAs are accompanied by addition of a long poly $(A)$ tail in the nucleus (Zhao et al., 1999). Following export to the cytoplasm, poly(A) binding protein (PABP) binds to the tail, thus stabilizing the mRNA and facilitating translation. Changes in poly(A) tail length occur throughout the lifetime of an mRNA; deadenylase enzymes shorten the poly(A) tails of mRNAs and play a key role in translational repression. In eukaryotes, degradation of normal cytoplasmic mRNA involves two general pathways, both of which begin with deadenylation (Fig. 1). In one pathway, deadenylation is followed by a decapping enzyme complex, DCP1-DCP2, which hydrolyzes the $5^{\prime}$ cap and exposes the mRNA body to $5^{\prime}-3^{\prime}$ digestion by XRN1. In the second pathway, deadenylation leads to a $3^{\prime}-5^{\prime}$ degradation of the RNA body by the exosome, which is a complex of $3^{\prime}-5^{\prime}$ exonucleases. The two pathways are not mutually exclusive, although the relative contributions of each are unclear.

In this review, we will focus on the multi-subunit and multifunctional CCR4-NOT complex and its role in deadenylation and mRNA degradation. We aim to summarize the current state of structure-function studies pertaining to the deadenylase components.

\section{STRUCTURE AND ASSEMBLY OF THE CCR4-NOT COMPLEX}

From earlier studies on the yeast complex by mass spectrometry and co-immunoprecipitation, Liu and colleagues were able to identify the existence of the CCR4-NOT complex approximately 0.9-1.2 MDa in size (Liu et al., 1998). The complex was named CCR4-NOT for the association of five NOT proteins, Not1p to Not5p, with Ccr4p and Pop2p. The complex is centered on the scaffold protein Not1p, which contains no known functional motifs but possesses two strikingly Glu-rich regions, and which is essential for yeast viability. Not4p has been identified to possess a functional RING finger domain in its N-terminal by NMR (Hanzawa et al., 2001), and its human homolog was subsequently confirmed to function as an E3 ligase by in vitro ubiquitination (Albert et al., 2002). Not2p contains no known functional motifs but has two functional domains: a C-terminal region involved in CCR4-NOT function, and an N-terminal domain that interacts with the protein Ada2 (Benson et al., 1998). Not3p and Not5p share similar $\mathrm{N}$-terminal regions, but their respective functions remain unclear. The complex also contains two deadenylases (to be discussed in more detail below), as well as Caf40p and Caf130p with indeterminate function. A number of binding partners have been identified for the $S$. cerevisiae

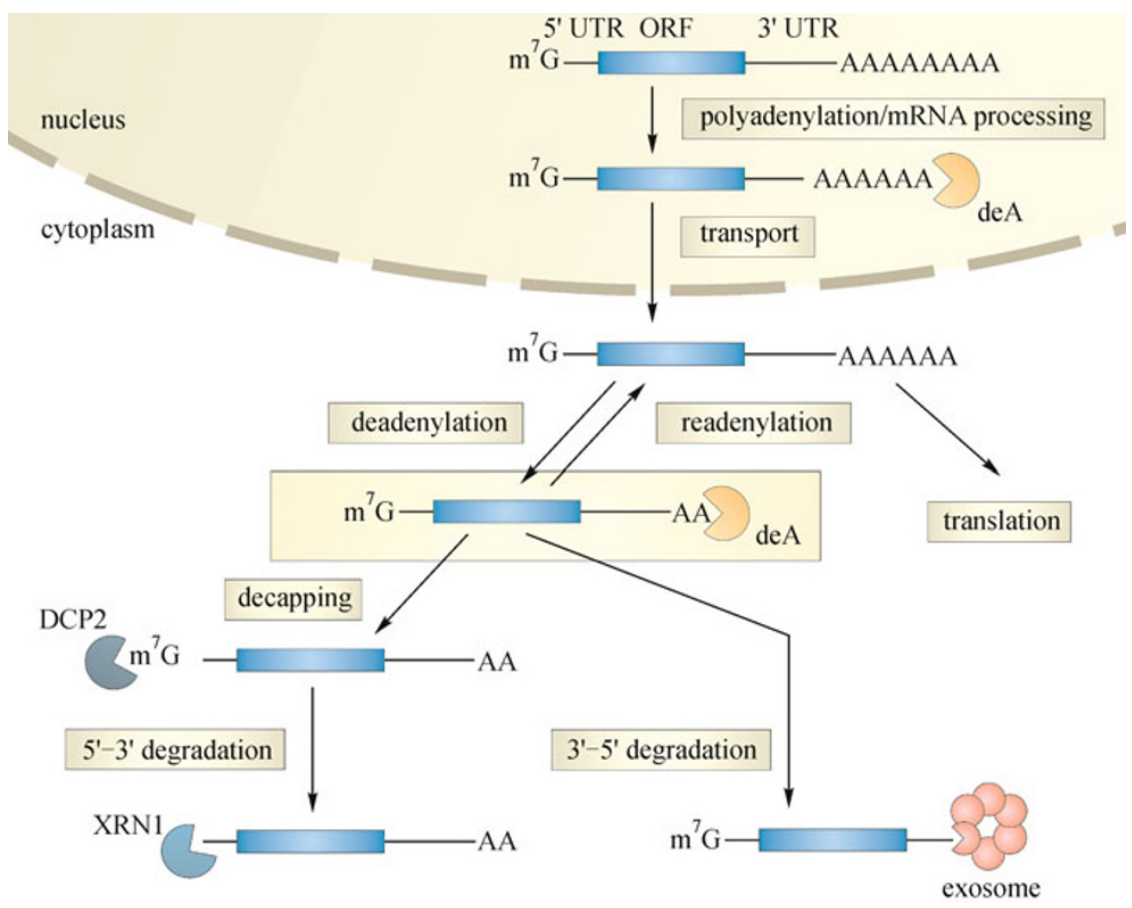

Figure 1. The two mRNA degradation pathways. Following the shortening of poly $(A)$ tails by deadenylase (deA) enzymes, the degradation of normal cytoplasmic mRNA involves two general pathways. In one pathway, deadenylation is followed by a decapping enzyme complex, DCP1-DCP2, which hydrolyzes the 5' cap and exposes the mRNA body to 5'-3' digestion by XRN1. In the second pathway, deadenylation leads to the $3^{\prime}-5^{\prime}$ degradation of the RNA body by the exosome. The cytoplasmic deadenylation step, in which the CCR4-NOT complex plays a major role, is highlighted. (Figure adapted from Goldstrohm and Wickens, 2008). 
complex but will not be discussed further in this review (see (Collart, 2003) for more details).

In contrast to the yeast CCR4-NOT complex, which is well understood as a result of yeast genetics studies, the human complex has been less well studied until recently. In 2009, Lau and colleagues studied the composition of the human CCR4-NOT complex from stable HeLa cell derivatives expressing epitope-tagged CCR4-NOT subunits (Lau et al., 2009). They determined the human complex to be generally similar to the yeast complex in composition, with CNOT1 as a scaffold and interacting with several CNOT proteins (CNOT2, CNOT7, CNOT8 and CNOT9). However, the authors also identified some differences between the yeast and human complexes, not least the presence of multiple deadenylases in human cells with different binding properties (to be discussed below). Furthermore, CNOT3 was not observed to bind directly to CNOT1 but instead binds to CNOT2 and may integrate into the complex via this interaction. Finally, CNOT4 is a true ortholog of yeast Not4p but, unlike its yeast counterpart, it appears not to be stably integrated into the human complex although it can interact with the scaffold protein CNOT1. A model can thus be proposed for the assembly of the human CCR4-NOT complex (Fig. 2).

\section{DEADENYLASES AND THEIR DIVERSITY}

Deadenylases, which constantly erode the poly $(A)$ tails of mRNA, are generally defined as magnesium-dependent exoribonucleases that recognize poly $(A)$ tails as their main substrate and hydrolyze RNA in the $3^{\prime}-5^{\prime}$ direction, resulting in release of $5^{\prime}$-AMP. Following removal of the poly(A) tail, other enzymes then initiate the degradation of mRNA. Deadenylation can occur both in the nucleus and the cytoplasm. In the nucleus, deadenylation restricts newly added poly $(A)$ tails of mRNA to their appropriate lengths, whereas extensive deadenylation of an mRNA in the cytoplasm initiates either degradation or repression. The deadenylation process can often be considered as a ratelimiting step for mRNA decay and translational silencing, and is therefore an ideal control point for both processes.

Biochemical and genetics analyses have greatly expanded the number of known deadenylases in recent years, and several putative deadenylases have been identified through bioinformatics but remain to be validated (see (Goldstrohm and Wickens, 2008) for a review). Deadenylases are currently categorized into two groups based on their nuclease domains. The first are the DEDD-type nucleases, so named after the conserved catalytic Asp and Glu residues in three exonuclease motifs. The major families of DEDD-type deadenylases include POP2, CAF1Z, poly(A)-specific ribonuclease (PARN) and PAN2. The second group is the exonuclease-endonuclease-phosphatase (EEP) family, which includes the families CCR4, Nocturnin, ANGEL and

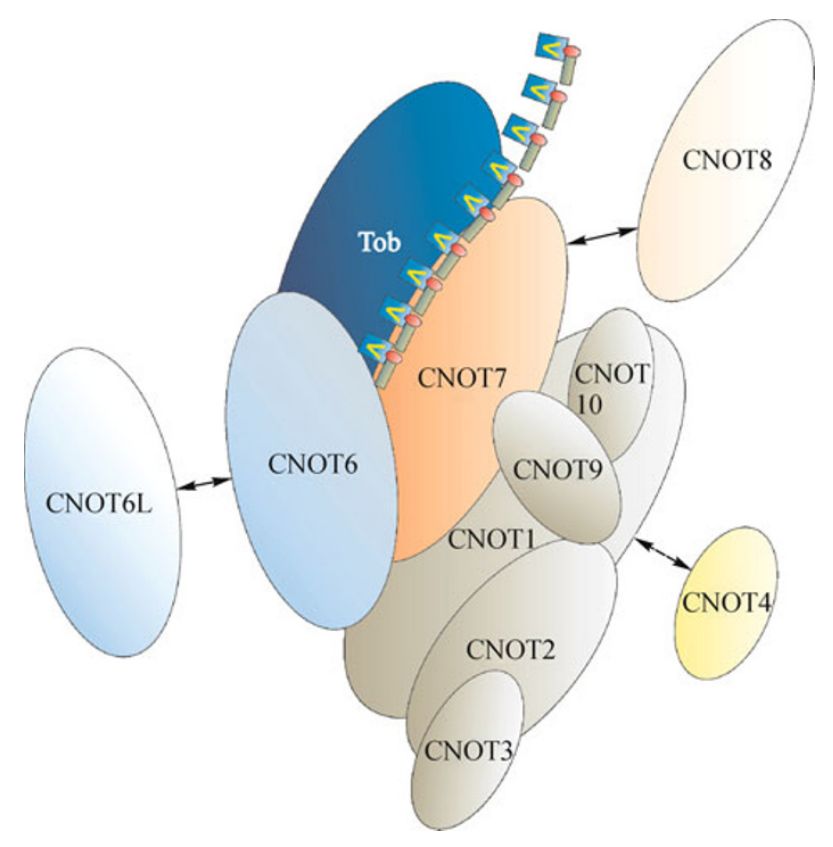

Figure 2. A model for the human CCR4-NOT complex. CNOT1 acts a scaffold to which the majority of the other CNOT proteins bind. CNOT3 is reported to have direct interaction with CNOT2 but not CNOT1. CNOT4 can bind CNOT1 is not stably associated with the CCR4-NOT complex. Human CCR4-NOT complexes are understood to only contain two deadenylases: one CCR4-type (either CNOT6 or CNOT6L) and one POP2type (CNOT7 or CNOT8). Tob can associate with the CCR4NOT complex via interaction with CNOT7. The CCR4-NOT proteins are labeled according to their name (i.e., CNOT1, CNOT2, etc.).

$2^{\prime}$ phosphodiesterase (2'PDE). The range of deadenylases varies among species, with members of the POP2, CCR4, PAN2 and ANGEL families present in all eukaryotes. Other deadenylases such as PAN and CAF1Z, which are both absent from Drosophila melanogaster, are less conserved. In addition to mRNA, poly(A) tails are also added to many nuclear, non-coding RNAs and are removed through deadenylation. The nuclear exosome complex for instance, has been strongly implicated in deadenylation; its RRP6 subunit is a DEDD-type nuclease and therefore the most likely deadenylating enzyme (Liu et al., 2006; Doma and Parker, 2007).

\section{THE CCR4-NOT DEADENYLASES}

The S. cerevisiae CCR4-NOT complex encompasses two deadenylases, Ccr4p and Pop2p (Caf1p), both of which are involved in mRNA degradation although Pop2p is dispensable for the deadenylase activity of Ccr4p. Human orthologs for most of the CCR4-NOT components in yeast have been 
identified. Interestingly, two orthologs have been identified for each of the yeast deadenylases, Ccr4p and Pop2p, in the human CCR4-NOT complex. The two human orthologs of yeast Ccr4p are CNOT6 (hCcr4a) and CNOT6L (hCcr4b), while the two orthologs of Pop2p are CNOT7 (hCAF1) and CNOT8 (hPOP2/CALIF). CNOT7 and CNOT8 are important for cell proliferation and share high amino acid sequence similarity with partially overlapping function. CNOT6 and CNOT6L, on the other hand, have different functions. CNOT7 and CNOT8 have been characterized as belonging to the DEDD-type nucleases, while CNOT6 and CNOT6L belong to the EEP superfamily.

A recent report suggests that the human CCR4-NOT complex contains variable deadenylase subunits (Lau et al., 2009). The authors showed the existence of distinct CCR4NOT complexes containing either CNOT7 or CNOT8. The implication is that CNOT7 and CNOT8 may compete for the same binding site on the scaffold protein CNOT1. A separate study by Aslam and colleagues showed that both CNOT7 and CNOT8 are important for efficient cell proliferation in MCF7 breast cancer cells. Furthermore, using knockdown of CNOT7 and CNOT8 genes by siRNA transfection, the authors showed that CNOT7 and CNOT8 have partial redundancy in cell proliferation and can compensate for each other's function, which is to be expected given their high amino acid sequence similarity. Microarray analysis, however, indicated that CNOT7 and CNOT8 also appear to have unique functions, and both CNOT7 and CNOT8 are known to have different substrate specificities for their enzymatic activity.

Lau and colleagues further reported that CNOT6 and CNOT6L may also be mutually exclusive in CCR4-NOT complexes, as they found no unique peptides of CNOT6L in purified CNOT6-tagged complexes (Lau et al., 2009). Earlier reports have suggested that CNOT6 and CNOT6L are functionally distinct: CNOT6L is required for cell proliferation via regulation of mRNA levels of the cell cycle inhibitor p27/ Kip1, whereas CNOT6 does not appear to be involved in cell proliferation (Morita et al., 2007). Interestingly, both CNOT6 and CNOT6L interact more tightly with CNOT7 than with CNOT8. In effect, the results from Lau and colleagues support a model whereby CCR4-NOT complexes are distinct and only contain a single Ccr4p ortholog (CNOT6 or CNOT6L) and a single Pop2p ortholog (CNOT7 or CNOT8).

The CCR4-NOT deadenylases have been shown to be important for a number of specific biologic processes. CNOT7, for instance, is implicated in both fertility and physiology. In the first case, Cnot7-knockout mice are defective in spermatogenesis and males are sterile, although the mice remain viable (Berthet et al., 2004; Nakamura et al., 2004). CNOT7 interacts with the retinoid $X$ receptor beta and therefore might serve as a coregulator of Rxrb in testicular somatic cells (Nakamura et al., 2004). In the second case, Cnot7-knockout mice exhibit increased bone mass as the result of enhanced bone formation, indicating that CNOT7 is an endogenous suppressor of bone mass (Washio-Oikawa et al., 2007). Furthermore, cell growth in mammals can be reduced by overexpression of CNOT7 or reduction of CNOT6L expression (Bogdan et al., 1998; Morita et al., 2007).

\section{STRUCTURE AND ACTIVITY OF THE POP2 DEADENYLASES}

The crystal structure of Pop2p was first determined from Saccharomyces cerevisiae in 2003 (Thore et al., 2003), and later from Schizosaccharomyces pombe in 2009 (Andersen et al., 2009) (Fig. 3). The overall structure of the S. cerevisiae Pop2p is kidney shaped with $13 \alpha$-helices and $6 \beta$-strands (Fig. 3A). Comparison with similar structures confirmed that Pop2 $p$ is a member of the DEDD-type nucleases with the common fold of the DnaQ subgroup. Despite low sequence similarity, Pop2p shares structural similarity with the $\epsilon$-subunit of DNA polymerase III and the exonuclease domain of DNA polymerase I. However, the $S$. cerevisiae Pop2p replaces the consensus DEDD nuclease motif with an SEDQ motif and so lacks several essential highly conserved catalytic residues, implying it may have lost its catalytic activity and that its role might be architectural. Pop2p was shown to have in vitro function as an active exonuclease on monotonous RNA sequences, with a slight preference for poly(A) RNA over poly $(U)$ and poly $(C)$. This lack of absolute specificity suggests that Pop2p may not bind RNA substrates in a specific manner, but may involve a substantial contribution of van der Waals interactions.

A structural and functional investigation of a homologous protein from Schizosaccharomyces pombe, or fission yeast, revealed more about the activity and selectivity of the deadenylase (Andersen et al., 2009). Unlike the S. cerevisiae Pop2p, its $S$. pombe counterpart does feature an intact DEDD motif (Fig. 3C). From the structure, two divalent metal ions (A and $B$ ) located in the active site were found to be essential for activity (Fig. 3B and 3C). Interestingly, although $\mathrm{Mg}^{2+}$ is found in higher concentrations inside cells, $S$. pombe Pop2p was found to prefer $\mathrm{Zn}^{2+}$ in the A site and $\mathrm{Mn}^{2+}$ in the $\mathrm{B}$ site (Fig. 3C), despite a large 100 -fold excess of $\mathrm{Mg}^{2+}$ in the cell. The identity of the ions in the active site was also found to have an effect on the kinetics of deadenylation. In the presence of $\mathrm{Mg}^{2+}, \mathrm{Mn}^{2+}$ and $\mathrm{Zn}^{2+}$ ions, deadenylation was slow and unspecific, and the authors observed only $\sim 25 \%$ of RNA substrates were completely deadenylated after $160 \mathrm{~min}$. In the absence of $\mathrm{Zn}^{2+}$, however, Pop2p quickly and specifically degraded the entire poly $(A)$ tail of the RNA substrate. This observation suggests that variations in the cellular $\mathrm{Zn}^{2+}$ levels might provide one means of regulating the overall rate of mRNA turnover.

The structure of human CNOT7, an ortholog of yeast 
A

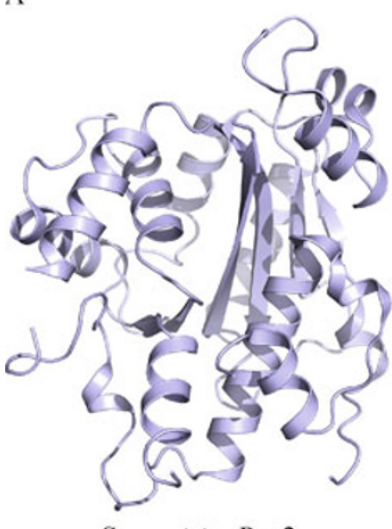

B

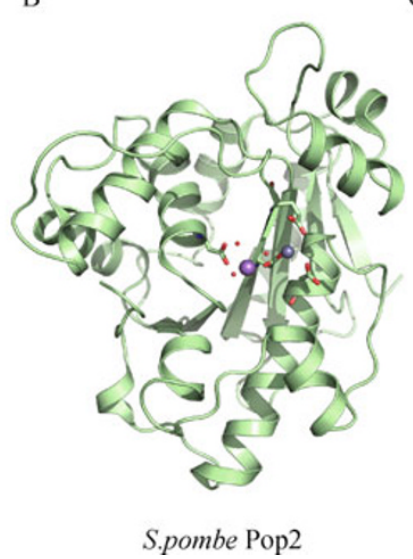

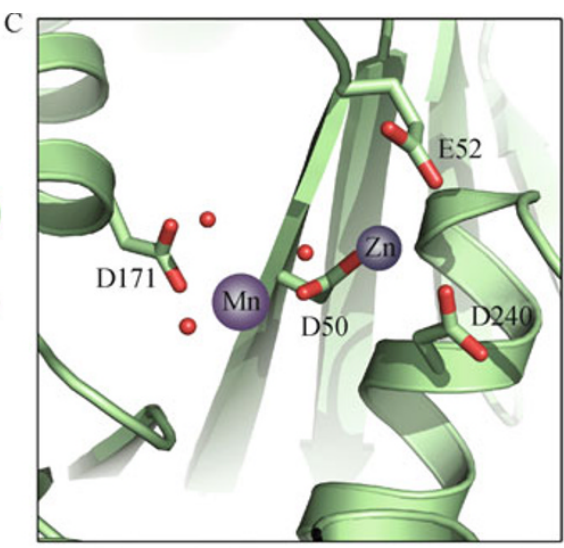

Figure 3. Crystal structures of yeast Pop2 enzymes. (A) The crystal structure of S. cerevisiae Pop2 (PDB ID: 1UOC), shown in pale blue. (B) The crystal structure of $S$. pombe Pop2 (PDB ID: 3G0Z), shown in pale green. (C) Enlarged view of the active site of $S$. pombe Pop2 with residues constituting the conserved DEDD motif shown in stick representation, $\mathrm{Zn}^{2+}$ and $\mathrm{Mn}^{2+}$ ions shown as spheres, and waters shown as small red spheres.

Pop2p, has been reported in complex with an antiproliferative protein Tob (Horiuchi et al., 2009). The interaction of Tob with CNOT7 will be discussed in more detail below. Unsurprisingly, CNOT7 was found to share the highest structural similarity to the related Pop2 from S. pombe and S. cerevisiae, as well as to a number of DEDD-type nucleases including human PARN. The structure of CNOT7 exhibited the core catalytic domain of the RNase D superfamily, characterized by the DEDD sequence motif. The conserved acidic amino acids are responsible for metal ion binding. As is the case for the $S$. pombe Pop2p structure, the authors of the human CNOT7 structure examined the nuclease activity of CNOT7 in the presence of the metal ions $\mathrm{Mg}^{2+}, \mathrm{Ca}^{2+}, \mathrm{Mn}^{2+}$ and $\mathrm{Co}^{2+}$. No activity was observed in the presence of $\mathrm{Ca}^{2+}$, and CNOT7 had significantly higher activity for RNA substrates over DNA substrates. Highest RNase activity was observed in the presence of $\mathrm{Mn}^{2+}$, suggesting that $\mathrm{Mn}^{2+}$ is required for full activity of CNOT7 (Horiuchi et al., 2009).

\section{THE CCR4 DEADENYLASES}

As a highly conserved member of the CCR4-NOT complex, $y C c r 4 p$ is the major cytoplasmic deadenylase in yeast and acts as the main catalytic component (Chen et al., 2002). Biochemical studies have placed the yeast Ccr4p in the EEP family, and it has been shown to contain three major functional domains (Draper et al., 1994). The N-terminal region is rich in glutamines and asparagines and is believed to have an activation domain that may interact with the transcriptional machinery. The central region contains several tandem copies of a leucine-rich-repeat (LRR) domain (Malvar et al., 1992), which has been shown to interact with yCaf1p (Draper et al., 1995), with other putative components of the core CCR4-NOT complex (Liu et al., 2001), and with potential binding ligands of the whole complex. This domain is thus considered to be the link that connects yCcr4p to the reminder of the complex (Draper et al., 1994; Liu et al., 1998) and other ligands. The LRR domain distinguishes all Ccr4p orthologs from other EEP family members and CCR4-like proteins (Dupressoir et al., 2001; Chen et al., 2002). The C-terminal region contains a deadenylase domain characteristic of the EEP superfamily with conserved catalytic Asp and His residues in the activation domain. The human orthologs, CNOT6 and CNOT6L, share the LRR domain and nuclease domain with $y C c r 4 p$, but lack the N-terminal Glu/Asp-rich region.

At the time of writing, no three-dimensional structure of a CCR4-type deadenylase has been determined. Nevertheless, within the EEP family, the C-terminal region of $y C c r 4 p$ exhibits significant homology to the exonuclease III group of proteins, also known as apurinic (AP) endonucleases, which are critical for maintaining genome integrity against the spontaneous production of abasic sites (Chen et al., 2002). Three representative AP endonucleases in this family are the exonuclease III (Exo III) from Escherichia coli, human APE1 (HAP1), and APN2 from S. cerevisiae (Chen et al., 2002). This family of $\mathrm{Mg}^{2+}$-dependent endonucleases is also homologous to inositol polyphosphate-5'-phosphatases and sphingomyelinases (Dlakić, 2000; Whisstock et al., 2000). From biochemical analysis, Chen and colleagues confirmed that yCcr4 displays $3^{\prime}-5^{\prime}$ exoribonuclease activity with strict poly $(A)$ substrate specificity and a preference toward substrates with at least two As at the $3^{\prime}$ end (Chen et al., 2002). 


\section{THE TOB/BTG ANTIPROLIFERATIVE PROTEINS}

The Tob/BTG family are antiproliferative proteins consisting of Tob, Tob2, BTG1, BTG2/Tis21/PC3, PC3B and BTG3/ANA in mammalian cells; AF177464 in Drosophila, and FOG-3 in Caenorhabiditis elegans. Tob/BTG proteins gain their antiproliferative activities due to their association with target proteins in cells, such as the association of Tob with the SMAD family to act as a negative regulator of SMAD signaling. Both Tob and BTG2 have been shown to interact with the CCR4-NOT complex via CNOT7 (Rouault et al., 1998; Prévôt et al., 2001), and BTG2 works as a co-activator of ERa-mediated transcription via a CCR4-like complex (Morel et al., 2003). To date, a number of crystal structures of Tob/BTG family members have been determined: human Tob1 (PDB ID: 2Z15), human BTG2 and mouse TIS21. A three-dimensional structure of the human Tob/CNOT7 complex has also been reported and will be discussed in further detail below. Tob, BTG2 and TIS21 share a similar structure comprised of five $\alpha$-helices and four $\beta$-strands that form two anti-parallel $\beta$-sheets. The $\mathrm{N}$-terminal leads immediately into a bundle of three $\alpha$-helices, followed by four $\beta$-strands with two small $\alpha$-helices inserted between strands $\beta 1$ and $\beta 2$.

The structure of human BTG2, which shares $40 \%$ sequence identity with Tob, shows three highly conserved domains among the Tob/BTG2 family (Fig. 4A) (Guéhenneux et al., 1997; Yang et al., 2008). Box A, also known as GR (for growth regulatory), is composed of strand $\beta 1$, the short helix $\alpha 3$, part of the $\alpha 2$ helix and a connecting loop between them. Two anti-parallel $\beta$-strands ( $\beta 2$ and $\beta 3$ ) form Box $B$, which is important for binding to a number of molecular targets including CNOT7. Box $C$ is composed of strand $\beta 4$ and the extended C-terminal loop. Helix $\alpha 1$, part of the $\alpha 2$ helix and the connecting loop between them constitute the putative HOXB9 interaction region. As BTG2 can associate with various molecular targets via different regions (Rouault et al., 1998; Prévôt et al., 2000; Berthet et al., 2002), the BTG2 structure showed that the relevant interfaces are located on different surfaces of BTG2 and may not interfere with each other, raising the possibility that BTG2 may be able to bind two or more molecular targets simultaneously in order to fulfil different regulatory requirements. Two $L X X L L$ motifs, also known as the nuclear receptor box (NR box), are located on helices $\alpha 2$ and $\alpha 5$, respectively. Interestingly, these two motifs are located on opposing faces of BTG2 and provide a hydrophilic surface, which might facilitate contact with nuclear receptors, while the hydrophobic residues are buried inside the core of the protein.

\section{THE INTERACTION OF TOB/BTG ANTIPROLIFERATIVE PROTEINS WITH CNOT7}

As mentioned above, accumulating evidence supports that the Tob/BTG family of proteins are common binding partners of CNOT7 in the CCR4-NOT complex. In 2009, Horiuchi and colleagues reported the crystal structure of the Tob-CNOT7 complex, thus demonstrating the mode of interaction between the two proteins, and suggested a mechanism for the antiproliferative activity of the complex (Horiuchi et al., 2009). The interaction of Tob with CNOT7 is largely hydrophobic and mediated by the conserved Box $A$ and Box $B$ regions (Fig. 4B). The presence of Tob had no appreciable effect on the activity of CNOT7. Based on their structure and functional
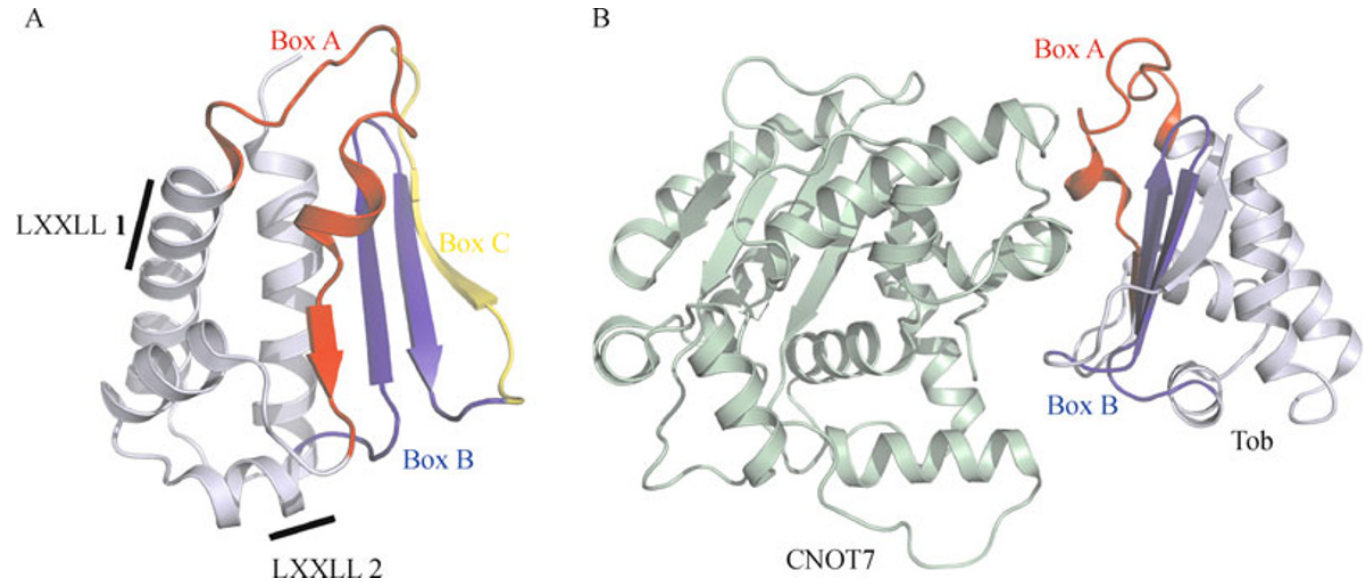

Figure 4. Structures of BTG2 and the Tob-CNOT7 complex. (A) The crystal structure of human BTG2 (PDB ID: 3DJU). The structure is shown in cartoon representation and colored pale blue. The conserved Box A region is colored red; the Box $B$ region colored blue; and the conserved Box $C$ region is colored yellow. Two conserved $L X X L L$ motifs are indicated. (B) The crystal structure of the human Tob-CNOT7 complex. The structure is shown in cartoon representation with CNOT7 colored pale green and Tob colored according to the scheme in panel A. 
analysis, Horiuchi and colleagues then proposed a hypothesis in which Tob recruits a PABP-mRNA complex to the CCR4-NOT complex via interaction with CNOT7, suggesting that CNOT7 functions as a bridge between Tob and the CCR4-NOT complex, rather than as a deadenylase. In their model, recruitment of the PABP-mRNA complex would cause translation to be interrupted by rapid degradation of the poly (A) tail, presumably by CNOT6 or CNOT6L, followed by repression of cell growth.

Analysis of human BTG2 by Yang and colleagues showed that it shares a significantly conserved CNOT7 binding interface with Tob (Yang et al., 2008). Moreover, functional analysis indicated that BTG2 suppresses CNOT7 deadenylase activity in vitro via direct interaction. However, structural analysis clearly showed that the binding interface of BTG2 and CNOT7 is not close to the active site of CNOT7, suggesting that binding of BTG2 may induce local conformational changes that influence the activity of CNOT7 or even distort the active site.

BTG2 was recently shown to be a general activator of mRNA degradation, for which the deadenylase activities of both CNOT7 and CCR4 are involved (Mauxion et al., 2008). The precise mechanism by which BTG2 enhances mRNA deadenylation remains to be determined, however, as BTG2 was unable to trigger the deadenylase activity of CNOT7 in vitro (Mauxion et al., 2008). In contrast, BTG2 was found to suppress the deadenylase activity of CAF1 in vitro (Yang et al., 2008), while Tob has also been shown to interact with the CCR4-NOT complex and inhibit its deadenylase activity in vitro (Miyasaka et al., 2008). The Tob/BTG family proteins therefore inhibit deadenylase activity associated with the CNOT7-containing CCR4-NOT complex, at least in vitro. CNOT7 alone is apparently not the decisive factor in mRNA deadenylation, and alternative pathways of cellular mRNA decay may come into play when its deadenylase activity is suppressed by BTG2. One possibility might be that CNOT7 may release its mRNA substrate and transfer it to other deadenylases such as PAN2-PAN3, although further studies are required to help understand the contradictory results for the roles of Tob and BTG2 on mRNA decay in vivo and in vitro.

\section{UNDERSTANDING DEADENYLATION AND MRNA DEGRADATION}

The presence of two deadenlyases in the CCR4-NOT complex remains puzzling and their mutual roles in mediating mRNA degradation is still unclear. Understanding the mutual functions of these two deadenylases, one belonging to the DEDD-type family and one to the EEP-type family, has been hampered in part by the lack of a three-dimensional structure for either yeast Ccr $4 p$ or its human orthologs. Data for the yeast CCR4-NOT complex has suggested that most of the nuclease activity is provided by Ccr4p (Chen et al., 2002; Tucker et al., 2002), and it may be the case that the role of Pop2p is to target the yeast CCR4-NOT complex to the poly (A) tail for further degradation by $\mathrm{Ccr} 4 \mathrm{p}$. The situation in the human CCR4-NOT complex is further complicated by the existence of two orthologs each for Pop2p and Ccr4p. The observation of four different complexes consisting of CNOT6CNOT7, CNOT6L-CNOT7, CNOT6-CNOT8 or CNOT6LCNOT8 heterodimers may provide an effective mechanism for the differential regulation of mRNA degradation (Lau et al., 2009). Further complexity is added by the association of the deadenylase heterodimers, consisting of one CCR4-type and one POP2-type protein, with other CNOT proteins in large multi-subunit "supercomplexes" with molecular weights ranging up to $2.0 \mathrm{MDa}$. It is not clear at this point what role, if any, the other CNOT proteins play in deadenylation.

In their study on the concerted action of poly $(\mathrm{A})$ nucleases, Yamashita and colleagues proposed that cytoplasmic deadenylation in mammalian cells is biphasic in nature (Yamashita et al., 2005). The first phase involves synchronous and stepwise shortening of the poly(A) tail to $\sim 110 \mathrm{nt}$. In the second phase, the mRNAs become more heterogeneous in the lengths of their poly(A) tails, ranging from $\sim 20 \mathrm{nt}$ to $\sim 110$ nt. The second phase is crucial to trigger decay of the mRNA body. Several observations support the hypothesis that biphasic deadenylation is the result of the sequential action of PAN2-PAN3 and CCR4-POP2 complexes (Fig. 5), with PAN2-PAN3 dominating the first phase and CCR4-POP2 the second phase. For instance, the poly $(A)$ binding protein (PABP) is reported to stimulate the PAN2-PAN3 complex but inhibits the CCR4-POP2 nuclease and DCP1-DCP2 decapping activities. Furthermore, changes in the activity of CCR4 affect the second phase of deadenylation, suggesting that the two phases are linked.

In summary, deadenylation is the first step in mRNA decay, and as such is an important biologic process with a number of important functions in the cell. The CCR4-NOT complex contains two deadenylases, yet efforts to understand their respective structures and activities have only scratched the surface. A number of important questions remain to be answered, such as how CCR4 and POP2 deadenylases cooperate to perform their functions. What role does the large CCR4-NOT complex play in the deadenylation process, and what are the factors that determine how mRNAs are regulated by specific deadenylases? Another important question to consider is how deadenylation is regulated. To date, a number of specific regulators that control deadenylation by recruiting CCR4-POP2 complexes have been reported, including PABP, PUF, CPEB, TTP and microRNAs (miRNAs). These latter miRNAs can elicit multifunctional control by repressing mRNA translation, enhancing mRNA decay and causing deadenylation, and are known to enhance mRNA deadenylation through the CCR4-NOT complex. PABP, for instance, is known to inhibit the deadenylase activity of the 


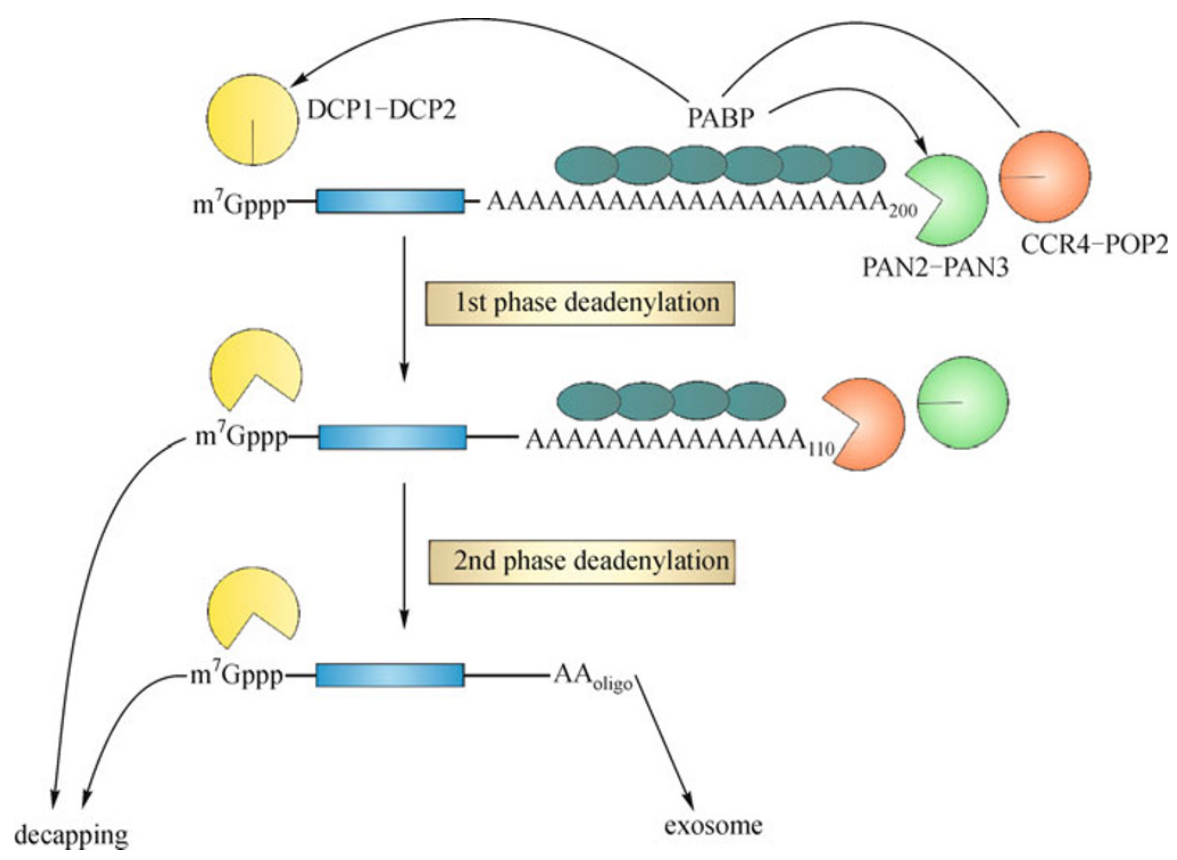

Figure 5. A model for the decay of cytoplasmic mRNA in mammalian cells. Cytoplasmic mRNA decay is reported to be a biphasic process involving the PAN2-PAN3 and CCR4-POP2 deadenylase complexes. PAN2-PAN3 is dominant in the first phase, in which poly $(A)$ tails are shortened to $\sim 110 \mathrm{nt}$. CCR4-POP2 is dominant in the second phase, which is crucial to initiate decay of the mRNA body (Figure adapted from Yamashita et al., 2005).

CCR4-NOT complex but not the PAN2-PAN3 deadenylase. It is evident that further structural and functional work is required to get to the heart of mRNA degradation, and to elucidate the roles of the multifunctional CCR4-NOT "supercomplex" in mRNA decay and beyond.

\section{ACKNOWLEDGMENTS}

This work was supported by the National Basic Research Program (973 Program) (Grant No. 2007CB914301) and the National Natural Science Foundation of China (Grant Nos. 30221003 and 30770438). This work was also supported in part by the Global COE Program (Integrative Life Science Based on the Study of Biosignaling Mechanisms), MEXT, Japan.

\section{ABBREVIATIONS}

AP, apurinic; EEP, exonuclease-endonuclease-phosphatase; Exo III, exonuclease III; HAP1,human APE1; LRR, leucine-rich-repeat; miRNAs, microRNAs; PABP, poly(A) binding protein; PARN, poly (A)-specific ribonuclease; NR box, receptor box; 2' PDE, 2' phosphodiesterase

\section{REFERENCES}

Albert, T.K., Lemaire, M., van Berkum, N.L., Gentz, R., Collart, M.A., and Timmers, H.T. (2000). Isolation and characterization of human orthologs of yeast CCR4-NOT complex subunits. Nucleic Acids Res 28, 809-817.
Albert, T.K., Hanzawa, H., Legtenberg, Y.I., de Ruwe, M.J., van den Heuvel, F.A., Collart, M.A., Boelens, R., and Timmers, H.T. (2002). Identification of a ubiquitin-protein ligase subunit within the CCR4NOT transcription repressor complex. EMBO J 21, 355-364.

Andersen, K.R., Jonstrup, A.T., Van, L.B., and Brodersen, D.E. (2009). The activity and selectivity of fission yeast Pop2p are affected by a high affinity for $\mathrm{Zn}^{2+}$ and $\mathrm{Mn}^{2+}$ in the active site. RNA 15, 850-861.

Badarinarayana, V., Chiang, Y.C., and Denis, C.L. (2000). Functional interaction of CCR4-NOT proteins with TATAA-binding protein (TBP) and its associated factors in yeast. Genetics 155, 1045-1054

Bai, Y., Salvadore, C., Chiang, Y.C., Collart, M.A., Liu, H.Y., and Denis, C.L. (1999). The CCR4 and CAF1 proteins of the CCR4NOT complex are physically and functionally separated from NOT2, NOT4, and NOT5. Mol Cell Biol 19, 6642-6651.

Benson, J.D., Benson, M., Howley, P.M., and Struhl, K. (1998). Association of distinct yeast Not2 functional domains with components of Gcn5 histone acetylase and Ccr4 transcriptional regulatory complexes. EMBO J 17, 6714-6722.

Berthet, C., Guéhenneux, F., Revol, V., Samarut, C., Lukaszewicz, A., Dehay, C., Dumontet, C., Magaud, J.P., and Rouault, J.P. (2002). Interaction of PRMT1 with BTG/TOB proteins in cell signalling: molecular analysis and functional aspects. Genes Cells 7, 29-39.

Berthet, C., Morera, A.M., Asensio, M.J., Chauvin, M.A., Morel, A.P., Dijoud, F., Magaud, J.P., Durand, P., and Rouault, J.P. (2004). CCR4-associated factor CAF1 is an essential factor for spermatogenesis. Mol Cell Biol 24, 5808-5820.

Bogdan, J.A., Adams-Burton, C., Pedicord, D.L., Sukovich, D.A., 
Benfield, P.A., Corjay, M.H., Stoltenborg, J.K., and Dicker, I.B. (1998). Human carbon catabolite repressor protein (CCR4)associative factor 1: cloning, expression and characterization of its interaction with the B-cell translocation protein BTG1. Biochem J 336, 471-481.

Chen, J., Rappsilber, J., Chiang, Y.C., Russell, P., Mann, M., and Denis, C.L. (2001). Purification and characterization of the $1.0 \mathrm{MDa}$ CCR4-NOT complex identifies two novel components of the complex. J Mol Biol 314, 683-694.

Chen, J., Chiang, Y.C., and Denis, C.L. (2002). CCR4, a 3-5 poly(A) RNA and ssDNA exonuclease, is the catalytic component of the cytoplasmic deadenylase. EMBO J 21, 1414-1426.

Collart, M.A. (2003). Global control of gene expression in yeast by the Ccr4-Not complex. Gene 313, 1-16.

Collart, M.A., and Struhl, K. (1993). CDC39, an essential nuclear protein that negatively regulates transcription and differentially affects the constitutive and inducible HIS3 promoters. EMBO J 12, 177-186.

Collart, M.A., and Struhl, K. (1994). NOT1(CDC39), NOT2(CDC36), NOT3, and NOT4 encode a global-negative regulator of transcription that differentially affects TATA-element utilization. Genes Dev 8, 525-537.

Collart, M.A., and Timmers, H.T. (2004). The eukaryotic Ccr4-not complex: a regulatory platform integrating mRNA metabolism with cellular signaling pathways? Prog Nucleic Acid Res Mol Biol 77, 289-322.

Cui, Y., Ramnarain, D.B., Chiang, Y.C., Ding, L.H., McMahon, J.S., and Denis, C.L. (2008). Genome wide expression analysis of the CCR4-NOT complex indicates that it consists of three modules with the NOT module controlling SAGA-responsive genes. Mol Genet Genomics 279, 323-337.

Deluen, C., James, N., Maillet, L., Molinete, M., Theiler, G., Lemaire, M., Paquet, N., and Collart, M.A. (2002). The Ccr4-not complex and yTAF1 (yTaf(II)130p/yTaf(II)145p) show physical and functional interactions. Mol Cell Biol 22, 6735-6749.

Denis, C.L., and Chen, J. (2003). The CCR4-NOT complex plays diverse roles in mRNA metabolism. Prog Nucleic Acid Res Mol Biol 73, 221-250.

Dlakić, M. (2000). Functionally unrelated signalling proteins contain a fold similar to $\mathrm{Mg}^{2+}$-dependent endonucleases. Trends Biochem Sci 25, 272-273.

Doma, M.K., and Parker, R. (2007). RNA quality control in eukaryotes. Cell 131, 660-668.

Draper, M.P., Liu, H.Y., Nelsbach, A.H., Mosley, S.P., and Denis, C.L. (1994). CCR4 is a glucose-regulated transcription factor whose leucine-rich repeat binds several proteins important for placing CCR4 in its proper promoter context. Mol Cell Biol 14, 4522-4531.

Draper, M.P., Salvadore, C., and Denis, C.L. (1995). Identification of a mouse protein whose homolog in Saccharomyces cerevisiae is a component of the CCR4 transcriptional regulatory complex. Mol Cell Biol 15, 3487-3495.

Dupressoir, A., Morel, A.P., Barbot, W., Loireau, M.P., Corbo, L., and Heidmann, T. (2001). Identification of four families of yCCR4- and $\mathrm{Mg}^{2+}$-dependent endonuclease-related proteins in higher eukaryotes, and characterization of orthologs of yCCR4 with a conserved leucine-rich repeat essential for $\mathrm{hCAF} 1 / \mathrm{hPOP} 2$ binding. BMC Genomics 2, 9.

Gavin, A.C., Bösche, M., Krause, R., Grandi, P., Marzioch, M., Bauer,
A., Schultz, J., Rick, J.M., Michon, A.M., Cruciat, C.M., et al. (2002). Functional organization of the yeast proteome by systematic analysis of protein complexes. Nature 415, 141-147.

Goldstrohm, A.C., and Wickens, M. (2008). Multifunctional deadenylase complexes diversify mRNA control. Nat Rev Mol Cell Biol 9, 337-344.

Guéhenneux, F., Duret, L., Callanan, M.B., Bouhas, R., Hayette, S., Berthet, C., Samarut, C., Rimokh, R., Birot, A.M., Wang, Q., et al. (1997). Cloning of the mouse BTG3 gene and definition of a new gene family (the BTG family) involved in the negative control of the cell cycle. Leukemia, 11, 370-375.

Hanzawa, H., de Ruwe, M.J., Albert, T.K., van Der Vliet, P.C., Timmers, H.T., and Boelens, R. (2001). The structure of the C4C4 ring finger of human NOT4 reveals features distinct from those of C3HC4 RING fingers. J Biol Chem 276, 10185-10190.

Hata, H., Mitsui, H., Liu, H., Bai, Y., Denis, C.L., Shimizu, Y., and Sakai, A. (1998). Dhh1p, a putative RNA helicase, associates with the general transcription factors Pop2 $p$ and Ccr4p from Saccharomyces cerevisiae. Genetics 148, 571-579.

Horiuchi, M., Takeuchi, K., Noda, N., Muroya, N., Suzuki, T., Nakamura, T., Kawamura-Tsuzuku, J., Takahasi, K., Yamamoto, T., and Inagaki, F. (2009). Structural basis for the antiproliferative activity of the Tob-hCaf1 complex. J Biol Chem 284, 13244-13255.

Komarnitsky, S.I., Chiang, Y.C., Luca, F.C., Chen, J., Toyn, J.H., Winey, M., Johnston, L.H., and Denis, C.L. (1998). DBF2 protein kinase binds to and acts through the cell cycle-regulated MOB1 protein. Mol Cell Biol 18, 2100-2107.

Lau, N.C., Kolkman, A., van Schaik, F.M., Mulder, K.W., Pijnappel, W. W., Heck, A.J., and Timmers, H.T. (2009). Human Ccr4-Not complexes contain variable deadenylase subunits. Biochem J 422, $443-453$.

Lee, T.I., Wyrick, J.J., Koh, S.S., Jennings, E.G., Gadbois, E.L., and Young, R.A. (1998). Interplay of positive and negative regulators in transcription initiation by RNA polymerase II holoenzyme. Mol Cell Biol 18, 4455-4462.

Lenssen, E., Oberholzer, U., Labarre, J., De Virgilio, C., and Collart, M.A. (2002). Saccharomyces cerevisiae Ccr4-not complex contributes to the control of Msn2p-dependent transcription by the Ras/cAMP pathway. Mol Microbiol 43, 1023-1037.

Liu, H.Y., Toyn, J.H., Chiang, Y.C., Draper, M.P., Johnston, L.H., and Denis, C.L. (1997). DBF2, a cell cycle-regulated protein kinase, is physically and functionally associated with the CCR4 transcriptional regulatory complex. EMBO J 16, 5289-5298.

Liu, H.Y., Badarinarayana, V., Audino, D.C., Rappsilber, J., Mann, M., and Denis, C.L. (1998). The NOT proteins are part of the CCR4 transcriptional complex and affect gene expression both positively and negatively. EMBO J 17, 1096-1106.

Liu, H.Y., Chiang, Y.C., Pan, J., Chen, J., Salvadore, C., Audino, D.C., Badarinarayana, V., Palaniswamy, V., Anderson, B., and Denis, C. L. (2001). Characterization of CAF4 and CAF16 reveals a functional connection between the CCR4-NOT complex and a subset of SRB proteins of the RNA polymerase II holoenzyme. J Biol Chem 276, 7541-7548.

Liu, Q., Greimann, J.C., and Lima, C.D. (2006). Reconstitution, activities, and structure of the eukaryotic RNA exosome. Cell 127, 1223-1237.

Mahadevan, S., and Struhl, K. (1990). Tc, an unusual promoter element required for constitutive transcription of the yeast HIS3 
gene. Mol Cell Biol 10, 4447-4455.

Maillet, L., and Collart, M.A. (2002). Interaction between Not1p, a component of the Ccr4-not complex, a global regulator of transcription, and Dhh1p, a putative RNA helicase. J Biol Chem 277, 2835-2842.

Malvar, T., Biron, R.W., Kaback, D.B., and Denis, C.L. (1992). The CCR4 protein from Saccharomyces cerevisiae contains a leucinerich repeat region which is required for its control of $A D H 2$ gene expression. Genetics 132, 951-962.

Mauxion, F., Faux, C., and Séraphin, B. (2008). The BTG2 protein is a general activator of mRNA deadenylation. EMBO $\mathrm{J} 27$, 1039-1048.

Miyasaka, T., Morita, M., Ito, K., Suzuki, T., Fukuda, H., Takeda, S., Inoue, J., Semba, K., and Yamamoto, T. (2008). Interaction of antiproliferative protein Tob with the CCR4-NOT deadenylase complex. Cancer Sci 99, 755-761.

Moqtaderi, Z., Bai, Y., Poon, D., Weil, P.A., and Struhl, K. (1996). TBPassociated factors are not generally required for transcriptional activation in yeast. Nature 383, 188-191.

Morel, A.P., Sentis, S., Bianchin, C., Le Romancer, M., Jonard, L., Rostan, M.C., Rimokh, R., and Corbo, L. (2003). BTG2 antiproliferative protein interacts with the human CCR4 complex existing in vivo in three cell-cycle-regulated forms. J Cell Sci 116, 2929-2936.

Morita, M., Suzuki, T., Nakamura, T., Yokoyama, K., Miyasaka, T., and Yamamoto, T. (2007). Depletion of mammalian CCR4b deadenylase triggers elevation of the p27Kip1 mRNA level and impairs cell growth. Mol Cell Biol 27, 4980-4990.

Nakamura, T., Yao, R., Ogawa, T., Suzuki, T., Ito, C., Tsunekawa, N., Inoue, K., Ajima, R., Miyasaka, T., Yoshida, Y., et al. (2004). Oligoastheno-teratozoospermia in mice lacking Cnot7, a regulator of retinoid X receptor beta. Nat Genet 36, 528-533.

Oberholzer, U., and Collart, M.A. (1998). Characterization of NOT5 that encodes a new component of the Not protein complex. Gene 207, 61-69.

Prévôt, D., Voeltzel, T., Birot, A.M., Morel, A.P., Rostan, M.C., Magaud, J.P., and Corbo, L. (2000). The leukemia-associated protein Btg1 and the p53-regulated protein Btg2 interact with the homeoprotein Hoxb9 and enhance its transcriptional activation. $J$ Biol Chem 275, 147-153.

Prévôt, D., Morel, A.P., Voeltzel, T., Rostan, M.C., Rimokh, R., Magaud, J.P., and Corbo, L. (2001). Relationships of the antiproliferative proteins BTG1 and BTG2 with CAF1, the human homolog of a component of the yeast CCR4 transcriptional complex: involvement in estrogen receptor alpha signaling path- way. J Biol Chem 276, 9640-9648.

Rouault, J.P., Prévôt, D., Berthet, C., Birot, A.M., Billaud, M., Magaud, J.P., and Corbo, L. (1998). Interaction of BTG1 and p53-regulated BTG2 gene products with mCaf1, the murine homolog of a component of the yeast CCR4 transcriptional regulatory complex. J Biol Chem 273, 22563-22569.

Seufert, W., and Jentsch, S. (1990). Ubiquitin-conjugating enzymes UBC4 and UBC5 mediate selective degradation of short-lived and abnormal proteins. EMBO J 9, 543-550.

Thore, S., Mauxion, F., Séraphin, B., and Suck, D. (2003). X-ray structure and activity of the yeast Pop2 protein: a nuclease subunit of the mRNA deadenylase complex. EMBO Rep 4, 1150-1155.

Tucker, M., Valencia-Sanchez, M.A., Staples, R.R., Chen, J., Denis, C.L., and Parker, R. (2001). The transcription factor associated Ccr4 and Caf1 proteins are components of the major cytoplasmic mRNA deadenylase in Saccharomyces cerevisiae. Cell 104, 377-386.

Tucker, M., Staples, R.R., Valencia-Sanchez, M.A., Muhlrad, D., and Parker, R. (2002). Ccr4p is the catalytic subunit of a Ccr4p/Pop2p/ Notp mRNA deadenylase complex in Saccharomyces cerevisiae. EMBO J 21, 1427-1436.

Viswanathan, P., Chen, J., Chiang, Y.C., and Denis, C.L. (2003). Identification of multiple RNA features that influence CCR4 deadenylation activity. J Biol Chem 278, 14949-14955.

Washio-Oikawa, K., Nakamura, T., Usui, M., Yoneda, M., Ezura, Y., Ishikawa, I., Nakashima, K., Noda, T., Yamamoto, T., and Noda, M. (2007). Cnot7-null mice exhibit high bone mass phenotype and modulation of BMP actions. J Bone Miner Res 22, 1217-1223.

Whisstock, J.C., Romero, S., Gurung, R., Nandurkar, H., Ooms, L.M., Bottomley, S.P., and Mitchell, C.A. (2000). The inositol polyphosphate 5-phosphatases and the apurinic/apyrimidinic base excision repair endonucleases share a common mechanism for catalysis. J Biol Chem 275, 37055-37061.

Yamashita, A., Chang, T.C., Yamashita, Y., Zhu, W., Zhong, Z., Chen, C.Y., and Shyu, A.B. (2005). Concerted action of poly(A) nucleases and decapping enzyme in mammalian mRNA turnover. Nat Struct Mol Biol 12, 1054-1063.

Yang, X., Morita, M., Wang, H., Suzuki, T., Yang, W., Luo, Y., Zhao, C., Yu, Y., Bartlam, M., Yamamoto, T., et al. (2008). Crystal structures of human BTG2 and mouse TIS21 involved in suppression of CAF1 deadenylase activity. Nucleic Acids Res 36, 6872-6881.

Zhao, J., Hyman, L., and Moore, C. (1999). Formation of mRNA 3 ends in eukaryotes: mechanism, regulation, and interrelationships with other steps in mRNA synthesis. Microbiol Mol Biol Rev 63, 405-445. 